Selected Papers . No. 16

\title{
Random Walks
}

in Stock-

Market Prices

By EUGENE F. FAMA

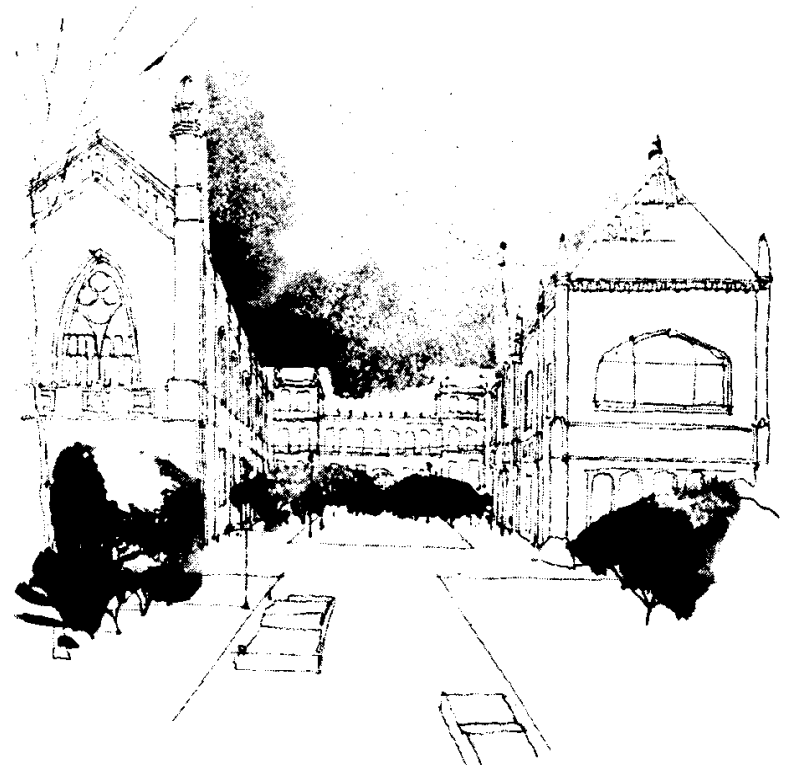

GRADUATE SCHOOL OF BUSINESS UNIVERSITY OF CHICAGO 
Eugene F. Fama is the Theodore 0. Yntema Professor of Finance at the Graduate School of Business of the University of Chicago. His research interests encompass the broad areas of economics, finance, statistics, mathematical methods, and computers, and he has been particularly concerned zoith the behavior of stock-market prices. A leader in developing the so-called "efficient markets" hypothesis, his influential writings have stimulated a large volume of related research at Chicago and elsewhere. Professor Fama received the B.A. degree (magna cum laude) from Tufts University in 1960 and the Ph.D. degree from the University of Chicago in 1964. His doctoral dissertation, "The Behavior of Stock-Market Prices," was published in the Journal of Business, January, 1965. It discussed the theory of random walks in substantial detail and provided extensive empirical evidence to support the theory. This Selected Paper-a condensed, nontechnical version of that article-initially was delivered as a talk at the 1965 Management Conference of the Graduate School of Business and the Executive Program Club. 


\section{Random Walks in}

\section{Stock- Market Prices}

FOR MANY YEARS economists, statisticians, and teachers of finance have been interested in developing and testing models of stock price behavior. One important model that has evolved from this research is the theory of random walks. This theory casts serious doubt on many other methods for describing and predicting stock price behavior-methods that have considerable popularity outside the academic world. For example, we shall see later that, if the random-walk theory is an accurate description of reality, then the various "technical" or "chartist" procedures for predicting stock prices are completely without value.

In general, the theory of random walks raises challenging questions for anyone who has more than a passing interest in understanding the behavior of stock prices. Unfortunately, however, most discussions of the theory have appeared in technical academic journals and in a form which the non-mathematician would usually find incomprehensible. This paper describes, briefly and simply, the theory of random walks and some of the important issues it raises concerning the work of market analysts. To preserve brevity, some aspects of the theory and its implications are omitted. More complete (but also more technical) discussions of the theory of random walks are available elsewhere; hopefully, the introduction provided here will encourage the reader to examine one of the more rigorous and lengthy works listed at the end of the paper. 


\section{Common Predictive Techniques}

In order to put the theory of random walks into perspective, we first discuss, in brief and general terms, the two approaches to predicting stock prices that are commonly espoused by market professionals. These are (1) "chartist" or "technical" theories and (2) the theory of fundamental or intrinsic value analysis.

The basic assumption of all the chartist or technical theories is that history tends to repeat itself, that is, past patterns of price behavior in individual securities will tend to recur in the future. Thus the way to predict stock prices (and, of course, increase one's potential gains) is to develop a familiarity with past patterns of price behavior in order to recognize situations of likely recurrence.

Essentially, then, chartist techniques attempt to use knowledge of the past behavior of a price series to predict the probable future behavior of the series. A statistician would characterize such techniques as assuming that successive price changes in individual securities are dependent. That is, the various chartist theories assume that the sequence of price changes prior to any given day is important in predicting the price change for that day.*

The techniques of the chartist have always been surrounded by a certain degree of mysticism, however, and as a result most market professionals have found them suspect. Thus it is probably safe to say that the pure chartist is relatively rare among stockmarket analysts. Rather the typical analyst adheres to a technique known as fundamental analysis or the intrinsic value method.

- Probably the best known example of the chartist approach to predicting stock prices is the Dow Theory. 
The assumption of the fundamental analysis approach is that at any point in time an individual security has an intrinsic value (or, in the terms of the economist, an equilibrium price) which depends on the earning potential of the security. The earning potential of the security depends in turn on such fundamental factors as quality of management, outlook for the industry and the economy, etc.

Through a careful study of these fundamental factors the analyst should, in principle, be able to determine whether the actual price of a security is above or below its intrinsic value. If actual prices tend to move toward intrinsic values, then attempting to determine the intrinsic value of a security is equivalent to making a prediction of its future price; and this is the essence of the predictive procedure implicit in fundamental analysis.

\section{Theory of Random Walks}

Chartist theories and the theory of fundamental analysis are really the province of the market professional and, to a large extent, of teachers of finance. Historically, however, there has been a large body of academic people, primarily economists and statisticians, who subscribe to a radically different approach to market analysis-the theory of random walks in stock-market prices. The remainder of this paper will be devoted to a discussion of this theory and its major implications.

Random-walk theorists usually start from the premise that the major security exchanges are good examples of "efficient" markets. An "efficient" market is defined as a market where there are large numbers of 
rational profit-maximizers actively competing, with each trying to predict future market values of individual securities, and where important current information is almost freely available to all participants.

In an efficient market, competition among the many intelligent participants leads to a situation where, at any point in time, actual prices of individual securities al ready reflect the effects of information based both on events that have already occurred and on events which as of now the market expects to take place in the future. In other words, in an efficient market at any point in time the actual price of a security will be a good estimate of its intrinsic value.

Now in an uncertain world the intrinsic value of a security can never be determined exactly. Thus there is always room for disagreement among market participants concerning just what the intrinsic value of an individual security is, and such disagree ment will give rise to discrepancies betwen actual prices and intrinsic values. In an efficient market, however, the actions of the many competing participants should cause the actual price of a security to wander randomly about its intrinsic value. If the discrepancies between actual prices and intrinsic values are systematic rather than random in nature, then knowledge of this should help intelligent market participants to better predict the path by which actual prices will move toward intrinsic values. When the many intelligent traders attempt to take advantage of this knowledge, however, they will tend to neutralize such systematic behavior in price series. Although uncertainty concerning intrinsic values will remain, actual prices of securities will wander randomly about their intrinsic values. 


\section{New Information}

Of course intrinsic values can themselves change across time as a result of new information. The new information may involve such things as the success of a current research and development project, a change in management, a tariff imposed on the industry's product by a foreign country, an increase in industrial production, or any other actual or anticipated change in a factor which is likely to affect the company's prospects.

In an efficient market, on the average, competition will cause the full effects of new information on intrinsic value to be reflected "instantaneously" in actual prices. In fact, however, because there is vagueness or uncertainty surrounding new information, "instantaneous adjustment" really has two implications. First, actual prices will initially overadjust to changes in intrinsic values as often as they will underadjust. Second, the lag in the complete adjustment of actual prices to successive new intrinsic values will itself be an independent, random variable, with the adjustment of actual prices sometimes preceding the occurrence of the event which is the basis of the change in intrinsic values (i.e., when the event is anticipated by the market before it actually occurs) and sometimes following.

This says that the "instantaneous adjustment" property of an efficient market implies that successive price changes in individual securities will be independent. $A$ market where successive price changes in individual securities are independent is, by definition, a random-walk market. Most simply the theory of random walks implies that a series of stock price changes has no memory-the past history of the series can- 
not be used to predict the future in any meaningful way. The future path of the price level of a security is no more predictable than the path of a series of cumulated random numbers.

It is unlikely that the random-walk hypothesis provides an exact description of the behavior of stock-market prices. For practical purposes, however, the model may be acceptable even though it does not fit the facts exactly. Thus, although successive price changes may not be strictly independent, the actual amount of dependence may be so small as to be unimportant.

What should be classified as unimportant depends, of course, on the question at hand. For the stock-market trader or investor the criterion is obvious: The independence assumption of the random-walk model is valid as long as knowledge of the past behavior of the series of price changes cannot be used to increase expected gains. More specifically, if successive price changes for a given security are independent, there is no problem in timing purchases and sales of that security. A simple policy of buying and holding the security will be as good as any more complicated mechanical procedure for timing purchases and sales. This implies that, for investment purposes, the independence assumption of the random-walk model is an adequate description of reality as long as the actual degree of dependence in series of pricechanges is not sufficient to make the expected profits of any more "sophisticated" mechanical trading rule or chartist technique greater than the expected profits under a naive buy-and-hold policy.

\section{Empirical Evidence on Independence}

Over the years a number of empirical tests of the random-walk theory have been 
performed; indeed, so many that it is not possible to discuss them adequately here. Therefore, in describing the empirical evidence, we limit ourselves to a brief discussion of different approaches employed and general conclusions that have evolved.

The main concern of empirical research on the random-walk model has been to test the hypothesis that successive price changes are independent. Two different approaches have been followed. First, there is the approach that relies primarily on common statistical tools such as serial correlation coefficients and analyses of runs of consecutive price changes of the same sign. If the statistical tests tend to support the assumption of independence, one then infers that there are probably no mechanical trading rules or chartist techniques, based solely on patterns in the past history of price changes, which would make the expected profits of the investor greater than they would be with a simple buy-and-hold policy. The second approach to testing independence proceeds by testing directly different mechanical trading rules to see whether or not they provide profits greater than buy-and-hold.

Research to date has tended to concentrate on the first or statistical approach to testing independence; the results have been consistent and impressive. I know of no study in which standard statistical tools have produced evidence of important de pendence in series of successive price changes. In general, these studies (and there are many of them) have tended to uphold the theory of random walks. This is true, for example, of the serial correlation tests of Cootner [4], Fama [ 5], Kendall [9], and Moore [10]. In all these studies" the sample serial correlation coefficients computed for

* See page 17. 
successive price changes were extremely close to zero, which is evidence against important dependence in the changes. Similarly, Fama's [5] analysis of runs of successive price changes of the same sign and the spectral analysis techniques of Granger and Morgenstern [ 8] and Godfrey, Granger, and Morgenstern [ 7] also support the independence assumption of the random-walk model.

We should emphasize, however, that, although the statistical techniques mentioned above have been the common tools used in testing independence, the chartist or technical theorist probably would not consider them adequate. For example, he would not consider either serial correlations or runs analyses as adequate tests of whether the past history of series of price changes can be used to increase the investor's expected profits. The simple linear relationships that underlie the serial correlation model are much too unsophisticated to pick up the complicated "patterns" that the chartist sees in stock prices. Similarly, the runs tests are much too rigid in their manner of determining the duration of upward and downward movements in prices. In particular, in runs-testing, a run is considered as terminated whenever there is a change in sign in the sequence of successive price changes, regardless of the size of the price change that causes the change in sign. The chartist would like to have a more sophisticated method for identifying movements-a method which does not always predict the termination of the movement simply because the price level has temporarily changed direction.

\section{Criticisms Heeded}

These criticisms of common statistical tools have not gone unheeded, however. For 
example, Alexander's filter technique [ 1, 2] is an attempt to apply more sophisticated criteria to the identification of moves. Although the filter technique does not correspond exactly to any well-known chartist theory, it is closely related to such things as the Dow Theory. Thus, the profitability of the filter technique can be used to make inferences concerning the potential profitability of other mechanical trading rules.

A filter of, say, 5 per cent is defined as follows: If the daily closing price of a particular security moves up at least 5 per cent, buy and hold the security until its price moves down at least 5 per cent from a subsequent high, at which time simultaneously sell and go short. The short position is maintained until the daily closing price rises at least 5 per cent above a subsequent low, at which time one should simultane ously cover and buy. Moves less than 5 per cent in either direction are ignored.

It is, of course, unnecessary to limit the size of the filter to 5 per cent. In fact, Professor Alexander has reported tests of the filter technique for filters ranging in size from 1 per cent to 50 per cent. The tests cover different time periods from 1897 to 1959 and involve daily closing prices for two indices, the Dow-Jones Industrials from 1897 to 1929 and Standard and Poor's Industrials from 1929 to 1959. In Alexander's latest work [2] it turns out that, even when the higher broker's commissions incurred under the filter rule are ignored, the filter technique cannot consistently beat the simple policy of buying and holding the indices for the different periods tested. Elsewhere I have tested the filter technique on individual securities. Again the simple buy-andhold method consistently beats the profits produced by different size filters. It seems, 
then, that, at least for the purposes of the individual trader or investor, tests of the filter technique also tend to support the random-walk model.

\section{Implications for Other Theories}

As stated earlier, chartist theories implicitly assume that there is dependence in series of successive price changes. That is, the history of the series can be used to make meaningful predictions concerning the future. On the other hand, the theory of random walks says that successive price changes are independent, that is, the past cannot be used to predict the future. Thus the two theories are diametrically opposed, and if, as the empirical evidence seems to suggest, the random-walk theory is valid, then chartist theories are akin to astrology and of no real value to the investor.

In an uncertain world, however, no amount of empirical testing is sufficient to establish the validity of a hypothesis beyond any shadow of doubt. The chartist or technical theorist always has the option of declaring that the evidence in support of the random-walk theory is not sufficient to validate the theory. On the other hand, the chartist must admit that the evidence in favor of the random-walk model is both consistent and voluminous, whereas there is precious little published discussion of rigorous empirical tests of the various technical theories. If the chartist rejects the evidence in favor of the random-walk model, his position is weak if his own theories have not been subjected to equally rigorous tests. This, I believe, is the challenge that the random-walk theory makes to the technician..

There is nothing in the above discussion, however, which suggests that superior fundamental or intrinsic value analysis is use 
less in a random-walk-efficient market. In fact, the analyst will do better than the investor who follows a simple buy-and-hold policy as long as he can more quickly identify situations where there are non-negligible discrepancies between actual prices and intrinsic values than other analysts and investors, and if he is better able to predict the occurrence of important events and evaluate their effects on intrinsic values.

If there are many analysts who are fairly good at this sort of thing, however, and if they have substantial resources at their disposal, they help narrow discrepancies between actual prices and intrinsic values and cause actual prices, on the average, to adjust "instantaneously" to changes in intrinsic values. That is, the existence of many sophisticated analysts helps make the market more efficient, which in turn implies a market which conforms more closely to the random-walk model. Although the returns to these sophisticated analysts may be quite high, they establish a market in which fundamental analysis is a fairly useless procedure both for the average analyst and for the average investor. That is, in a randomwalk-efficient market, on the average, a security chosen by a mediocre analyst will produce a return no better than that obtained from a randomly selected security of the same general riskiness.

\section{A Natural Benchmark}

There probably are not many analysts (in fact, I know of none) who would willingly concede that they are no better than the "average" analyst. If all analysts think they are better than average, however, this only means that their estimate of the average is biased downward. Fortunately, it is not 
necessary to judge an analyst solely by his claims. The discussion above provides a natural benchmark with which we can evaluate his performance.

In a random-walk-efficient market at any point in time the market price of a security will al ready reflect the judgments of many analysts concerning the relevance of currently available information to the prospects of that security. Now an individual analyst may feel that he has better insights than those that are already implicit in the market price. For example, he may feel that a discrepancy between market price and intrinsic value exists for some security, or he may think the intrinsic value of the security is itself about to change because of some impending piece of new information which is not yet generally available.

These "insights" of the analyst are of no real value, however, unless they are eventually borne out in the market, that is, unless the actual market price eventually moves in the predicted direction. In other words, if the analyst can make meaningful judgments concerning the purchase and sale of individual securities, his choices should consistently outperform randomly selected securities of the same general riskiness. It must be stressed, however, that the analyst must consistently produce results better than random selection, since, by the nature of uncertainty, for any given time period he has about a 50 per cent chance of doing better than random selection even if his powers of analysis are completely nonexistent. Moreover, not only must the analyst do consistently better than random selection, but he must beat random selection by an amount which is at least sufficient to cover the cost of the resources (including his own time) which are expended in the process of carry- 
ing out his more complicated selection procedures.

What we propose, then, is that the analyst subject his performance to a rigorous comparison with a random selection procedure. One simple practical way of comparing the results produced by an analyst with a random selection procedure is the following: Every time the analyst recommends a security for purchase (or sale), another security of the same general riskiness is chosen randomly. A future date is then chosen at which time the results produced by the two securities will be compared. Even if the analyst is no better than the random selection procedure, in any given comparison there is still 50 per cent chance that the security he has chosen will outperform the randomly selected security. After the game has been played for a while, however, and the results of many different comparisons are accumulated, then it will become clear whether the analyst is worth his salt or not.

\section{Comparing Portfolios}

In many circumstances, however, the primary concern is with the performance of a portfolio rather than with the performance of individual securities in the portfolio. In this situation one would want to compare the performance of the portfolio in question with that of a portfolio of randomly selected securities. A useful benchmark for randomly selected portfolios has been provided by Fisher and Lorie [6]. They computed rates of return for investments in common stocks on the New York Stock Exchange for various time periods from 1926 to 1960 . The basic assumption in all their computations is that at the beginning of each period studied the investor puts an 
equal amount of -money into each common stock listed at that time on the Exchange. This amounts to random sampling where the sampling is, of course, exhaustive. Different rates of return are then computed for different possible tax brackets of the investor, first under the assumption that all dividends are reinvested in the month paid, and then under the assumption that dividends are not reinvested.

A possible procedure for the analyst is to compare returns for given time periods earned by portfolios he has managed with the returns earned for the same time periods by the Fisher-Lorie "randomly selected" portfolios. It is important to note, however, that this will be a valid test procedure only if the portfolios managed by the analyst had about the same degree of riskiness as the Fisher-Lorie "market" portfolios. If this is not the case, the Fisher-Lorie results will not provide a proper benchmark. In order to make a proper comparison between the results produced by the analyst and a random selection policy, it will be necessary to define and study the behavior of portfolios of randomly selected securities, where these portfolios are selected in such a way that they have about the same degree of riskiness as those managed by the analyst.

If the claims of analysts concerning the advantages of fundamental analysis have any basis in fact, the tests suggested above would seem to be easy to pass. In fact, however, the only "analysts" that have so far undergone these tests are open-end mutual funds. In their appeals to the public, mutual funds usually make two basic claims: (1) Because it pools the resources of many individuals, a fund can diversify much more effectively than the average, small investor; and (2) because of its management's close 
ness to the market, the fund is better able to detect "good buys" in individual securities. In most cases the first claim is probably true. The second, however, implies that mutual funds provide a higher return than would be earned by a portfolio of randomly selected securities. In a separate paper [5] I reported the results of a study which suggest that, if the initial loading charges of mutual funds are ignored, on the average the funds do about as well as a randomly selected portfolio. If one takes into account the higher initial loading charges of the funds, however, on the average the random investment policy outperforms the funds. In addition, these results would seem to be consistent with those of the now famous Wharton study of mutual funds [ 11].

These adverse results with respect to mutual funds have tended to lead random-walk theorists to feel that other financial institutions, and most professional investment advisers as well, probably do no better than random selection. Institutions and analysts can only dispel such doubts by submitting their performance to a rigorous comparison with a random selection procedure.

\section{Conclusion}

In sum the theory of random walks in stock-market prices presents important challenges to both the chartist and the proponent of fundamental analysis. For the chartist, the challenge is straightforward. If the random-walk model is a valid description of reality, the work of the chartist, like that of the astrologer, is of no real value in stockmarket analysis. The empirical evidence to date provides strong support for the random-walk model. In this light the only way the chartist can vindicate his position is to show that he can consistently use his tech- 
niques to make better-than-chance predictions of stock prices. It is not enough for him to talk mystically about patterns that he sees in the data. He must show that he can consistently use these patterns to make meaningful predictions of future prices.

The challenge of the theory of random walks to the proponent of fundamental analysis, however, is more involved. If the random-walk theory is valid and if security exchanges are "efficient" markets, then stock prices at any point in time will represent good estimates of intrinsic or fundamental values. Thus, additional fundamental analysis is of value only when the analyst has new information which was not fully considered in forming current market prices, or has new insights concerning the effects of generally available information which are not already implicit in current prices. If the analyst has neither better insights nor new information, he may as well forget about fundamental analysis and choose securities by some random selection procedure.

In essence, the challenge of the randomwalk theory to the proponent of fundamental analysis is to show that his more complicated procedures are actually more profitable than a simple random selection policy. As in the case of the chartist, the challenge is an empirical one. The analyst cannot merely protest that he thinks the securities he selects do better than randomly selected securities; he must demonstrate that this is in fact the case. 


\section{REFERENCES}

1. Alexander, Sdney S. "Price Movements in Speculative Markets: Trends or Random Walks,"

(May, 1961), 7-26.

2. ---. "Price Movements in Speculative Markets: Trends or Random Walks, Number 2," ibid., V (Spring, 1964), 25-46.

3. Cootner, Paul H. (ed). The R andom Character of Stock Market Prices. Cambridge: M.I.T. Press, 1964. An excellent compilation of re search on the theory of random walks completed prior to mid-1963.

4. Cootner, Paul H. "Stock Prices: Random vs. Systematic Changes," Industrial Management III, (Spring, 1962), 24-45.

5. Fama, Eugene F. "The Behavior of Stock-Market Prices," Journal of Business, XXXVIII (January, 1965), 34-105.

6. FISHER, L., and LORIE, J. H. "Rates of Return on Investments in Common Stocks," Journal of Business, XXXVJI (January, 1964), 1-21.

7. Godfrey, Michael D., Grancer, Clive W. J., and MORGENSTERN, OSKAR. "The Random Walk Hypothesis of Stock Market Behavior," Kyklos, XVII (January, 1964), I-30.

8. Granger, Clive W. J., and Morgenstern, 0. "Spectral A nalysis of N ew York Stock Market Prices," Kyklos, XVI (January, 1963), I-27.

9. KendalL, M. G. "The Analysis of Economic Time Series," Journal of the R oyal Statisticol Society (Series A), XCVI (1953), 11-25.

10. Moore, Arnold. "A Statistical Analysis of Common-Stock Prices." Unpublished Ph.D. dissertation, Graduate School of Business, University of Chicago (1962).

11. "A Study of Mutual Funds." Prepared for the Securities and Exchange Commission by the Wharton School of Finance and Commerce. Report of the Committee on Interstate and Foreign Commerce. Washington, DC.: Government Printing Office, 1962. 